\title{
The Potential of Magnetic Resonance Urography in the Diagnosis of ureteral Strictures
}

DO1. 10, 17691/stm2018, 10.4.14

Received January 25, 2018

E.S. Davydova, Radiologist, Magnetic Resonance Imaging Unit, Department of Radiology';

E.A. Egorova, MD, DSc, Professor, Department of Diagnostic Radiology²

${ }^{1}$ Branch Clinical Diagnostic Center of PJSC Gazprom, 16 Nametkin St., Moscow, 117997, Russia;

${ }^{2}$ A.I. Yevdokimov Moscow State University of Medicine and Dentistry, 20 Delegatskaya St., 127473, Moscow, Russia

The aim of the study was to assess the sensitivity of magnetic resonance urography (MRU) - both native and contrast-enhanced examinations - in the diagnosis of ureteral strictures and to evaluate its importance in the diagnostic algorithm in patients with different causes of urinary obstruction.

Materials and Methods. MRU was performed in 62 patients with ureteral strictures, whose average age was $47 \pm 9.8$ years, using high-field MRU devices Vantage Atlas (Toshiba, Japan) and Ingenia (Philips, the Netherlands) with magnetic field intensities of 1.5 and 3.0 T, respectively. The strictures were primary in 6 cases and secondary (acquired) in 56 . In 42 cases (67.7\%), excretory MRU was carried out: the procedure involved intravenous administration of contrast agent. The detected strictures were evaluated in terms of localization, shape, extension and suprastenotic dilatation extent. The technique sensitivity in detecting the changes in surrounding structures was also estimated: ureteral wall thickening and paraureteral tissue infiltration were evaluated, the spread of tumor process was assessed when appropriate. The results obtained by computed tomography urography (CTU) and confirmed by surgical intervention were considered as reference values.

Results. MRU sensitivity in the diagnosis of ureteral strictures was $96.3 \%$. The technique showed the best results in the lower third of the ureters: $100 \%$ of strictures were identified regardless of their origin. In the upper third, MRU confirmed the strictures detected on CTU in $97.2 \%$ of cases. As for the middle third of the organ, the results were lower than on CTU: $87.4 \%$ of strictures were detected. According to excretory MRU data, the following pathological symptoms were revealed: organ obstruction - in 62 cases $(100 \%)$, filling defect — in 9 cases $(14.5 \%)$, suprastenotic dilatation - in 56 cases $(90.3 \%)$, hydronephrosis - in 52 cases $(83.9 \%)$, pelvicalyceal system dilation in 58 cases $(93.5 \%)$. Besides, traditional MR sequences, especially the series with suppression of the signal from adipose tissue, are highly sensitive to edema manifestations and revealed infiltrative changes in the surrounding tissue in $34(54.8 \%)$ and ureteral wall thickening in 45 cases $(72.5 \%)$. Accumulation of fluid in the pelvis was found in 8 cases $(12.9 \%)$. MRU also made it possible to detect changes in parenchymal organs, the liver, adrenal glands and to suspect the presence of secondary pelvic bone lesions.

Conclusion. Contrast-enhanced MRU demonstrates high efficacy in detection of ureteral strictures similarly to CTU and, in case of obstruction located in the lower third of the organ, it is superior to CTU and provides additional information about the condition of the organ wall and the surrounding tissues. Non-contrast MRU provides the possibility to clearly localize the level of obstruction and identify its cause. The absence of ionizing radiation makes the technique successfully applicable in patients with contraindications to CTU.

Key words: magnetic resonance urography; ureteral stricture; obstruction; hydronephrosis.

\section{Introduction}

A ureteral stricture is a pathological narrowing of the ureteral lumen, usually causing an obstruction to the outflow of urine from the renal pelvis. The ureteral obstruction revealed on radiological examination may have benign or malignant nature and be due to extraand intra-luminal changes. Statistical data presented by the Federal State Statistics Service in the report on morbidity rates of the main disease classes among the

Corresponding author: Ekaterina S. Davydova, e-mail: davydova_ekaterina@yahoo.com 
population (2012) show that the incidence of urogenital pathologies has remained unchanged in recent years and amounted to $11,718.7$ per 100 thousand people over the past 5 years. Unfortunately, there is no separate registry of ureteral diseases in the Russian Federation.

There are primary (congenital) and secondary (acquired) obstructions. Primary obstructions include congenital obstructing megaureter, e.g. scarring of the ureteral wall due to existing genetic abnormalities or compression of the ureter when crossed by blood vessels. Acquired strictures are numerous. Based on the obstruction cause, they may be divided into intrinsic and extrinsic. Intrinsic strictures may be caused by oncological disease (primary urothelial cancer), follow urinary infection (tuberculosis, gonorrhea) or passage of calculi. Extrinsic strictures are formed as a result of compression of the ureter by an external pathological process (mass lesions in the pelvis, enlarged lymph nodes, including those resulting from retroperitoneal fibrosis, with fibrous tissue growth and development of one- or two-sided compression of the ureter). Secondary involvement of ureters (compression, invasion) occurs in pelvic tumors 6 times more often than primary tumors [1-2]. According to the etiology, it is also possible to distinguish non-iatrogenic and iatrogenic obstruction, whose frequency varies widely: $0.05-30 \%$ depending on the surgeon's experience and technical complexity of surgery [3-6]. latrogenic ureteral injuries may be the result of both open surgery and laparoscopic or endoscopic procedures and often are not recognized during the operation itself, therefore statistically the percentage of their occurrence in literature can be reduced $[4,7]$.

Ureteral injuries during gynecological and obstetric interventions in women are due to the abundance of vascular neoplasms and close anatomical relationship between the internal reproductive organs and the urinary tract. The frequency of ureteral injury resulting from gynecologic surgery in the pelvic cavity is estimated at between 0.3 and $1 \%[6,7]$. Usually the ureter is damaged during gynecologic surgery for benign and malignant neoplasms of the uterus and ovaries, iatrogenic injuries to the upper urinary tract are caused by hysterectomy in $54 \%$ of cases [7-10]. Kachrilos from the Royal London Hospital [11] proposed his own classification of ureteral strictures based on the nature of the stricture and involvement of the ureteral lumen (Table 1).

Today, the widespread technique of excretory urography $(E U)$ is used less often due to its restrictions and numerous cases of ambiguous results and the gold standard for diagnosis of ureteral strictures and other causes of urinary obstruction appears to be computed tomography urography (CTU). This examination is a quick and revealing way to assess the state of the urinary tract $[12,13]$. However, in some patients the use of ionizing radiation is undesirable. These include individuals requiring multiple dynamic examinations (especially the young), pregnant women, patients with impaired renal function and persons with allergic reactions to contrast agent. In these situations, fast developing magnetic resonance urography (MRU) may become a method of choice making it possible to carry out comprehensive examination of the entire urinary tract similarly to CTU.

Magnetic resonance examination of the urinary tract can be performed in two ways: by means of static noncontrast urography using ultrafast T2-weighted images (T2-WI) like those applied in magnetic resonance cholangiopancreatography and using T1-WI after intravenous administration of contrast agent (similar to X-ray EU). Non-contrast MRU technique is based on obtaining a high-intensity magnetic-resonance signal from motionless or slow-moving fluid located in natural and/or pathological structures of the examined area, the signal from the surrounding tissues being much less intense. T2-WI images in the frontal plane are the basic sequence for static MRU, they allow clear visualization of the urinary tract with its narrowing, cysts of different localization, the vertebral canal.

Today, MRU is a fast developing technique. A number

Table 1

Classification of ureteral strictures based on the cause of their appearance and involvement of the ureteral lumen (by S. Kachrilas et al., 2013)

\begin{tabular}{|c|c|c|}
\hline Strictures & External compression & Lumen narrowing \\
\hline Malignant & $\begin{array}{l}\text { Gynecological malignancies } \\
\text { Colorectal cancer } \\
\text { Cancer-associated lymphadenopathy } \\
\text { Primary/secondary retroperitoneal malignancy }\end{array}$ & $\begin{array}{l}\text { Transitional cell carcinoma of the ureter } \\
\text { Pelvic tumors (prostate, bladder) }\end{array}$ \\
\hline Benign & $\begin{array}{l}\text { Idiopathic retroperitoneal fibrosis } \\
\text { Endometriosis }\end{array}$ & $\begin{array}{l}\text { Infection (tuberculosis) } \\
\text { Traumatic injury } \\
\text { latrogenic injury } \\
\text { Ureteral intestinal anastomosis } \\
\text { Kidney transplantation } \\
\text { Effects of radiation therapy/chemotherapy } \\
\text { Ureterolithiasis }\end{array}$ \\
\hline
\end{tabular}


of studies have demonstrated its high efficacy in detecting the level of urinary obstruction [12, 14, 15]. However, the potential of the method in identifying the causes of urinary tract abnormalities remains understudied [16].

The aim of the study was to assess the sensitivity of magnetic resonance urography (both native and contrast-enhanced examinations) in the diagnosis of ureteral strictures and to evaluate its importance in the diagnostic algorithm in patients with different forms of urinary obstruction.

\section{Materials and Methods}

A total of 62 patients with the upper urinary tract abnormalities underwent complex radiological examination at the City Clinical Hospital named after S.I. Spasokukotsky, City Clinical Hospital named after S.S. Yudin and the Branch Clinical Diagnostic Center of PJSC Gazprom (Moscow) over the period from 2013 to 2017. The examination included EU, ultrasound, dynamic CTU and MRU.

The study complies with the Declaration of Helsinki (2013) and was performed following approval by the Ethics Committee of A.I. Yevdokimov Moscow State University of Medicine and Dentistry. Written informed consent was obtained from every patient.

Of 62 patients with ureteral disorders included in the study, 6 patients $(9.7 \%)$ had primary strictures, 56 $(90.3 \%)$ - secondary (acquired). The mean age of patients was $47 \pm 9.8$ years. The distribution of patients under study is shown in Figure 1.

Magnetic resonance urography was performed using high-field devices Vantage Atlas (Toshiba, Japan) and Ingenia (Philips, the Netherlands) with magnetic field intensities of 1.5 and $3.0 \mathrm{~T}$, respectively. A larger FOV index was applied to visualize the entire ureter. Patients were administered Buscopan (120 mg) or Drotaverine (120 mg) per os $30 \mathrm{~min}$ prior to the examination to reduce the number of motor artifacts and intravenous

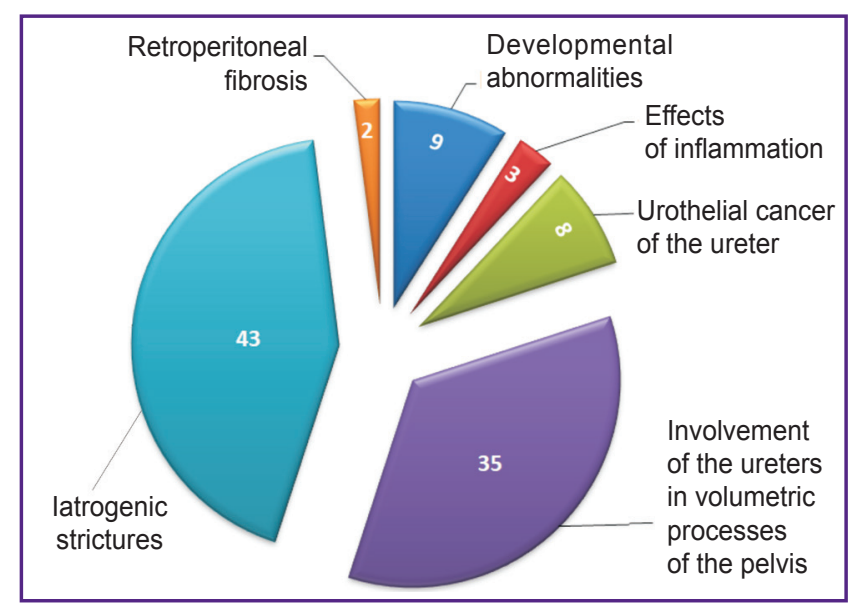

Figure 1. The pattern of ureteral changes (\%) diuretic in the form of Furosemide $(10 \mathrm{mg}) 15 \mathrm{~min}$ before the procedure. In 42 cases $(67.7 \%)$, excretory MRU was performed: the procedure involved intravenous administration of contrast agent (Gadovist $-0.1 \mathrm{mmol} /$ $\mathrm{kg}$ body weight), in 20 patients (32.3\%), it was limited to static native examination alone.

The detected strictures were evaluated in terms of their localization, shape, extension and suprastenotic dilatation extent. The technique sensitivity in detecting changes in the surrounding structures was also evaluated: ureteral wall thickening, paraureteral tissue infiltration and the spread of tumor process when appropriate. CTU results confirmed by surgical intervention were considered as reference values.

\section{Results}

MRU sensitivity in the diagnosis of ureteral strictures amounted to $96.3 \%$. The technique demonstrated the highest results in the lower third of the ureter: $100 \%$ of strictures were identified regardless of their origin. In the upper third, MRU confirmed the strictures revealed by CTU in $97.2 \%$ of cases. As for the middle third of the organ, the results were lower than those obtained by CTU: 52 strictures (83.9\%) were revealed. In 44 cases $(71.0 \%)$, the localization of strictures was also confirmed by cystoscopy and subsequent surgery: 27 patients $(43.5 \%)$ underwent Boari flap ureteroplasty, 17 patients (27.4\%) underwent Da Vinci robot-assisted ureteroureteroanastomosis (Intuitive Surgical, USA). In 18 cases $(29 \%)$, ureteral bougienage followed by stenting was performed, partial patency of the organ preserved.

Static MRU showed enlarged upper urinary tract characterized by a hyperintensive magnetic resonance signal on T2-WI: due to a high signal from the urine, the kidneys, ureters, and bladder were visualized simultaneously. T2-WI with thick slices allowed obtaining images in different planes and reconstruct MIP images from them. In 11 cases (17.7\%), excretory MRU revealed and clarified the boundaries of a soft tissue component of the existing tumor not visualized in non-contrast series. In 4 cases, accumulation of contrast agent by the thickened walls of the organ and surrounding cellular tissue infiltration confirmed the assumption about the infectious process.

According to MRU data, the following signs of urinary tract disease were revealed: ureteral obstruction $(n=62 ; 100 \%)$, filling defect $(n=9 ; 14.5 \%)$, suprastenotic dilation $(n=56 ; 90.3 \%)$, hydronephrosis $(n=52 ; 83.9 \%)$, pelvicalyceal system dilation $(n=58 ; 93.5 \%)$ (Table 2$)$. Stricture length varied from 11 to $83 \mathrm{~mm}$, the lumen width of the narrowed ureter - from 0.8 to $3.6 \mathrm{~mm}$ (the normal width of the ureter is $4-7 \mathrm{~mm}$ ).

In 2 cases $(3.2 \%)$, small volume formations of the ureter (up to $3 \mathrm{~mm}$ ) were overlooked by MRU, probably due to the limited spatial resolution, these formations were detected during dynamic observation, CT and were confirmed by biopsy. 
Ureteral wall thickening of various length was found in 23 cases $(37.1 \%)$, that was a manifestation of the inflammatory process during dynamic observation in 4 patients, it accompanied primary urothelial cancer of the ureter in 3 patients and cicatricial changes of the wall due to strictures in 16 cases (26.7\%). The software capabilities enabled effective image post-processing and ascertaining ultrasound and CT data (Figure 2).

Besides, MRU provided additional information on the surrounding organs and tissues in $18.2 \%$ of cases as compared to CTU. Static MRU revealed infiltrative changes in the surrounding cellular tissue in 28 patients $(45.2 \%)$ due to high sensitivity of traditional magnetic
Table 2

The signs of ureteral pathology according to the data obtained by radiology and other verification methods $(n=62)$ (abs. number)

\begin{tabular}{|c|c|c|c|c|}
\hline Sign & EU & US & MRU & $\begin{array}{c}\text { Dynamic } \\
\text { CTU }\end{array}$ \\
\hline Filling defect & 7 & - & 9 & 11 \\
\hline Retention changes in the upper urinary tract & 49 & 50 & 52 & 52 \\
\hline Surrounding tissue infiltration & - & 21 & 28 & 28 \\
\hline Ureteral wall thickening & - & - & 23 & 23 \\
\hline Delay in contrast agent excretion & 10 & - & 8 & 8 \\
\hline
\end{tabular}

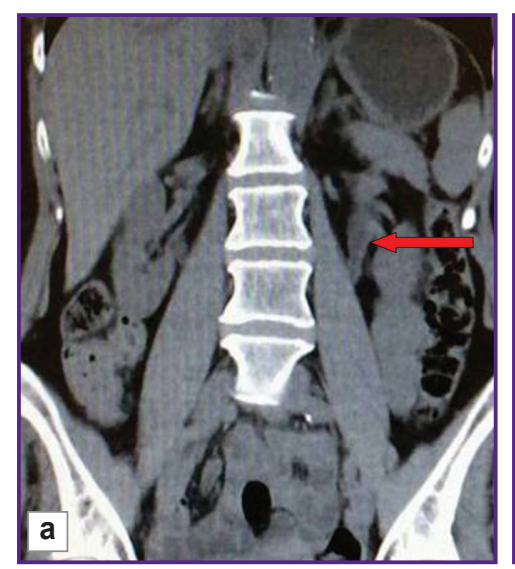

resonance sequences and especially the series with suppression of the signal from adipose tissue.

Accumulation of fluid in the pelvis was detected in 8
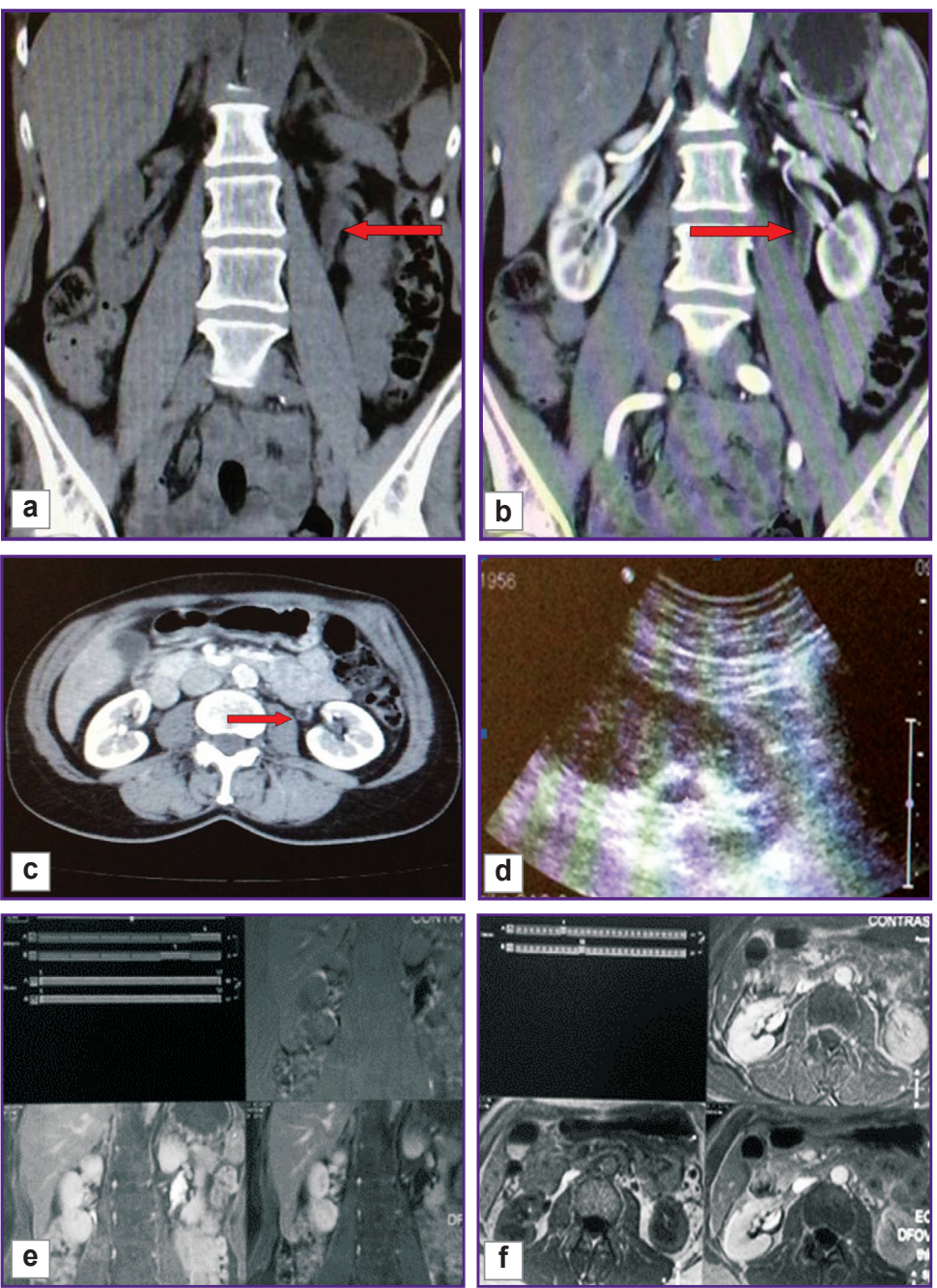

Figure 2. Ureteral pathology revealed in patient $F$. (aged 58) by different radiology methods:

CTU of the abdominal cavity in the coronal (a), (b) and axial (c) projections: (a) in the native phase of the examination there is left ureteropelvic wall thickening (weakly hyperdense in relation to urine) (arrow); (b), (c) early diffuse mural accumulation of contrast agent at the level of this thickening (arrows); (d) an echogram of the left kidney: only dilated calices are visualized; post-processed MRU images in the coronal (e) and axial (f) planes: a "map" of direct contrast agent accumulation by left ureteropelvic junction walls (the image marked CONTRAST) as a result of subtraction of image (b) from image (a) 

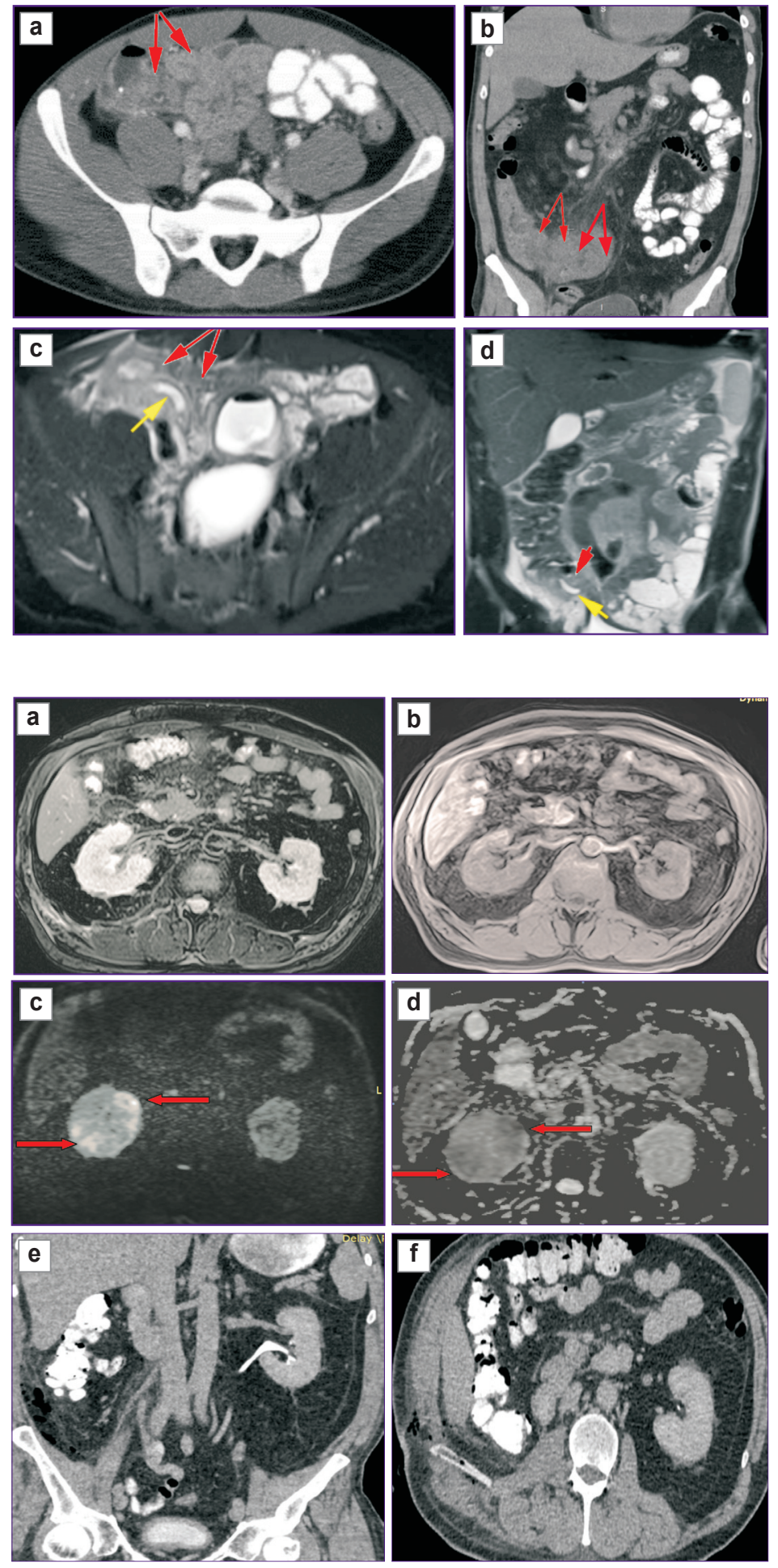

Figure 4. Patient S., 42 years, with intraoperative ureteral trauma: MRU: modes T2 Fat Sat (a) and post-contrast T1-WI (b) the changes in the images are not convincingly visualized; DWI (c) and ADC (d) clearly defined areas of restricted diffusion corresponding to apostemes (arrows); CTU in the coronal (e) and axial (f) planes after removal of the right kidney

patients $(12.9 \%)$. The effusion was equally well detected both on magnetic resonance images and during ultrasound due to high sensitivity of both methods.
Figure 3. The results of examination of patient $\mathrm{K}$. (aged 35) admitted with a diagnosis of "right-sided renal colic":

CTU in the axial (a) and coronal (b) planes: the enlarged cecum and ileum with inflammatory changes in the surrounding tissue are defined in the right iliac region (red arrows); MRU of the abdominal cavity (T2-WI with suppression of the signal from adipose tissue) in the axial (c) and coronal (d) planes: there is an enlarged and fluid filled appendix (yellow arrow), infiltrated changes of cecum and distal ileum walls, the adjacent omentum and paracolic adipose tissue (red arrows), a small amount of free fluid in the cecum

The results of tomographic examination led to the conclusion about the presence of acute appendicitis, local peritonitis, omentitis, typhlitis, terminal ileitis
Standard T2-WI and sequences with suppression of the signal from adipose tissue enabled visualizing fluid accumulations and differentiating them from such formations as ovarian cysts and follicles.

When there were areas of local pain as well as clinical and laboratory manifestations of inflammation, CTU and MRU were the most informative in differentiating the diagnosis of urinary obstruction from other diseases. CTU data were used to define the presence of calculi, the level of ureteral obstruction, perifocal infiltration in the cellular tissue, the signs of intestinal obstruction, the presence of free and localized fluid and gas accumulations in the abdominal cavity and retroperitoneal space. When using T1-WI and T2-WI in the axial and coronal planes (including sequences with suppression of the signal from adipose tissue as well as diffusion-weighted images), MRU provided additional possibility to reveal even the minimum volume of reactive effusion, changes in the walls of the source organ, syntopic organs and the surrounding tissue (Figure 3 ).

MRU also made it possible to detect complications of ureteral surgery, such as pyelonephritis, in 8 patients $(12.9 \%)$. In 4 patients, the information obtained by MRU was unique. For example, in 2 patients, the use of DWI-MRU enabled finding small liquid accumulations in the cortical substance of the kidney which were not visualized on CTU (including that with contrast-enhancement). These areas were classified as manifestations of apostematous pyelonephritis and were the reason for changing the management of patients: the affected kidneys were removed (Figure 4). Besides, magnetic 
Figure 5. The results of radiology examination of patient L., 29 years, at pregnancy week $36-37$ :

(a) echogram: hypoechogenic areas with fuzzy contours detected in the right kidney (arrows) enabled suspecting inflammatory changes, another hypoechogenic structure (not marked) corresponds to a simple cyst; MRU: (b) T2-WI in the sagittal plane, the uterus is enlarged in accordance with the term of pregnancy, in the uterine cavity there is one fetus in cephalic presentation; (c) image Co 3D MRCP, no convincing data on the presence of calculi have been revealed, the signal from the pelvicalyceal system and the ureter is weak due to weak current of fluid inside of them; (d), (e) modes DWI and $A D C$, areas of restricted diffusion indicate the regions of purulent inflammation (arrows)
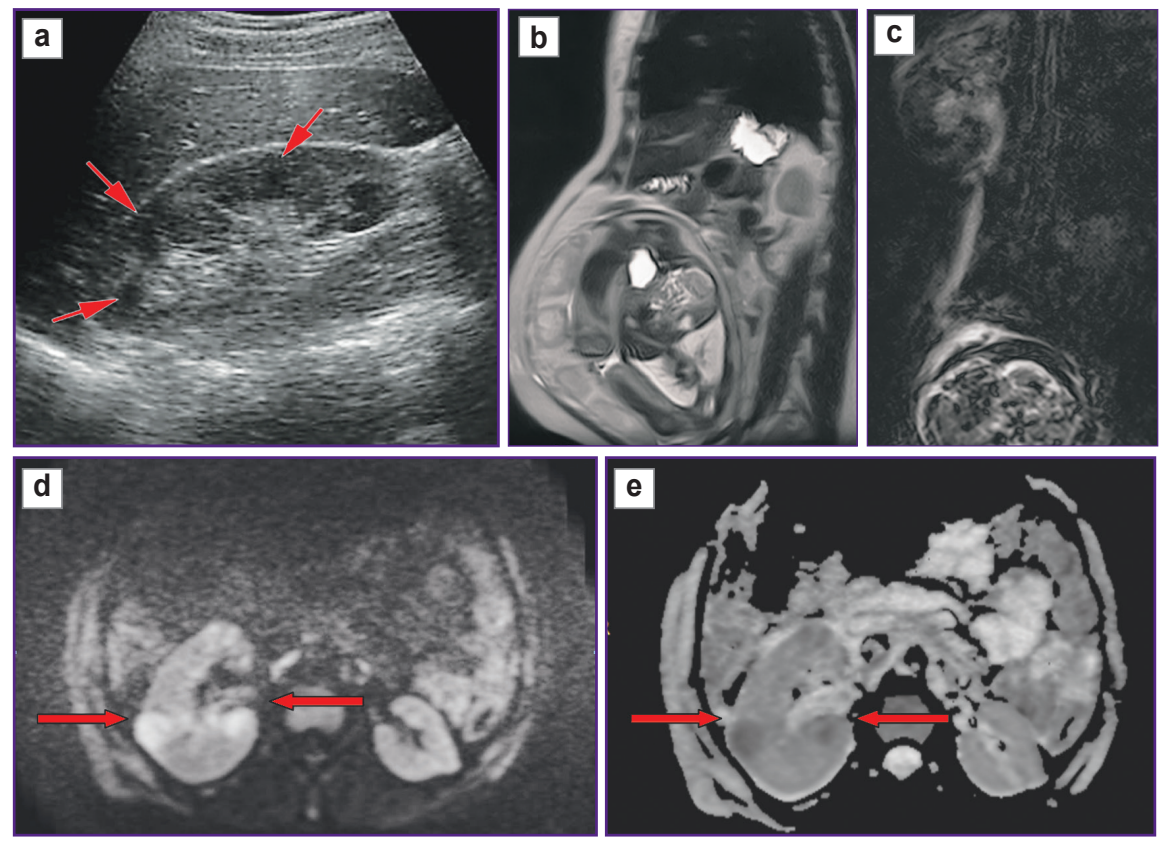

resonance examination revealed purulent inflammation of the kidneys in 2 pregnant patients for whom it was impossible to undergo CTU and ultrasound could only help suspect the cause of fever (Figure 5).

MRU also revealed changes in parenchymal organs (the kidneys, liver, pancreas, adrenal glands) and the presence of secondary pelvic bone lesion that was found in 17 cases $(27.4 \%)$.

\section{Conclusion}

Magnetic resonance urography and computed tomography urography have similar diagnostic efficacy in detecting ureteral strictures of different genesis. It is possible in both cases to make a full comprehensive single-step evaluation of the urinary tract as a whole. At the same time, magnetic resonance urography provides the possibility to obtain additional diagnostic information about the condition of the organ wall and surrounding tissue infiltration. The possibility to evaluate the abdominal and pelvic organs comprehensively enables us to rule out other pathology and determine the tactics of patient management.

The absence of ionizing radiation makes magnetic resonance urography the method of choice in examination of pregnant women, children, and patients with impaired renal function. High diagnostic efficacy of the method makes it applicable for dynamic control of treatment results in persons of different age groups.

Study funding. This study was not supported by any financial sources.

Conflict of interests. The authors declare that the study, its subject and content do not involve conflicting interests.

\section{References}

1. Gupta R., Paner G.P., Amin M.B. Neoplasms of the upper urinary tract: a review with focus on urothelial carcinoma of the pelvicalyceal system and aspects related to its diagnosis and reporting. Adv Anat Pathol 2008; 15(3): 127-139, https:// doi.org/10.1097/pap.0b013e31817145a9.

2. Paner G.P., Zehnder P., Amin A.M., Husain A.N., Desai M.M. Urothelial neoplasms of the urinary bladder occurring in young adult and pediatric patients: a comprehensive review of literature with implications for patient management. Adv Anat Pathol 2011; 18(1): 79-89, https://doi. org/10.1097/pap.0b013e318204c0cf.

3. Palaniappa N.C., Telem D.A., Ranasinghe N.E., Divino C.M. Incidence of iatrogenic ureteral injury after laparoscopic colectomy. Arch Surg 2012; 147(3): 267-271, https://doi.org/10.1001/archsurg.2011.2029.

4. Delacroix S.E. Jr., Winters J.C. Urinary tract injures: recognition and management. Clin Colon Rectal Surg 2010; 23(2): 104-112, https://doi.org/10.1055/s-0030-1254297.

5. Vasilyeva M.A., Egorova E.A. Capabilities of ultrasound study and computed tomography in the diagnosis of circumscribed peritonitis in ureteral perforation. Vestnik rentgenologii i radiologii 2011; 2: 55-58.

6. Parpala-Spårman T., Paananen I., Santala M., Ohtonen P., Hellström P. Increasing numbers of ureteric injuries after the introduction of laparoscopic surgery. Scan J Urol Nephrol 2008; 42(5): 422-427, https://doi. org/10.1080/00365590802025857.

7. Klap J., Phé V., Chartier-Kastler E., Mozer P., Bitker M.O., Rouprêt M. Aetiology and management of iatrogenic injury of the ureter: a review. Prog Urol 2012; 22(15): 913-919, https://doi.org/10.1016/j.purol.2012.05.003.

8. Siram S.M., Gerald S.Z., Greene W.R., Hughes K., Oyetunji T.A., Chrouser K., Cornwell E.E. 3rd, Chang D.C. Ureteral trauma: patterns and mechanisms of injury of an uncommon condition. Am J Surg 2010; 199(4): 566-570, https://doi.org/10.1016/j.amjsurg.2009.11.001. 
9. Loran O.B., Seregin A.V., Dovlatov Z.A. Surgical treatment of iatrogenic strictures and obliterations in the pelvic ureter in women. Eksperimentalnaya $i$ klinicheskaya urologiya 2015; 3: 128-131.

10. Esparaz A.M., Pearl J.A., Herts B.R., LeBlanc J., Kapoor B. latrogenic urinary tract injuries: etiology, diagnosis, and management. Semin Intervent Radiol 2015; 32(2): 195208, https://doi.org/10.1055/s-0035-1549378.

11. Kachrilas S., Bourdoumis A., Karaolides T., Nikitopoulou S., Papadopoulos G., Buchholz N., Masood J. Current status on minimally invasive endoscopic management of ureteric strictures. Ther Adv Urol 2013; 5(6): 354-365, https://doi.org/10.1177/1756287213505671.

12. Silverman S.G., Leyendecker J.R., Amis E.S. Jr. What is the current role of CT urography and MR urography in the evaluation of the urinary tract? Radiology 2009; 250(2): 309232, https://doi.org/10.1148/radiol.2502080534.

13. Rouprêt M., Zigeuner R., Palou J., Boehle A., Kaasinen E., Sylvester R., Babjuk M., Oosterlinck W.
European guidelines for the diagnosis and management of upper urinary tract urothelial cell carcinomas: 2011 update. Eur Urol 2011; 59(4): 584-594, https://doi.org/10.1016/j. eururo.2010.12.042.

14. Ergen F.B., Hussain H.K., Carlos R.C., Johnson T.D., Adusumilli S., Weadock W.J., Korobkin M., Francis I.R. 3D excretory MR urography: improved image quality with intravenous saline and diuretic administration. J Magn Reson Imaging 2007; 25(4): 783-789, https://doi.org/10.1002/ jmri.20875.

15. Blandino A., Minutoli F., Gaeta M., Settineri N., Pandolfo I. MR pyelography in the assessment of hydroureteronephrosis: single-shot thick-slab RARE versus multislice HASTE sequences. Abdom Imaging 2013; 28(3): 433-439, https://doi.org/10.1007/s00261-002-0054-y.

16. Takahashi N., Kawashima A., Glockner J.F., Hartman R.P., Kim B., King B.F. MR urography for suspected upper tract urothelial carcinoma. Eur Radiol 2009; 19(4): 912923, https://doi.org/10.1007/s00330-008-1228-y. 